\title{
Kernos
}

Revue internationale et pluridisciplinaire de religion grecque antique

2| 1989

Varia

\section{À propos des déesses Maliades et de quelques épiclèses gréco-asianiques}

\section{René Lebrun}

\section{(apenEdition \\ Journals}

\section{Édition électronique}

URL : http://journals.openedition.org/kernos/238

DOI : $10.4000 /$ kernos. 238

ISSN : 2034-7871

\section{Éditeur}

Centre international d'étude de la religion grecque antique

\section{Édition imprimée}

Date de publication : 1 janvier 1989

Pagination : 83-88

ISSN : 0776-3824

\section{Référence électronique}

René Lebrun, «À propos des déesses Maliades et de quelques épiclèses gréco-asianiques », Kernos [En ligne], 2 | 1989, mis en ligne le 02 mars 2011, consulté le 05 mai 2019. URL : http:// journals.openedition.org/kernos/238; DOI : 10.4000/kernos.238 
Kernos, 2 (1989), p. 83-88.

\section{À PROPOS DES DÉESSES MALIADES ET DE QUELQUES ÉPICLÈSES GRÉCO-ASIANIQUES}

\section{Les divinités Maliades}

Les $M \alpha \lambda t \alpha ́ \delta \varepsilon \varsigma$ sont notamment mentionnées dans le Philoctète de Sophocle :

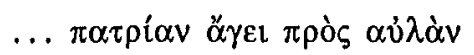

$\mathrm{M} \alpha \lambda t \alpha \dot{\delta} \delta \omega v v v \mu \varphi \hat{\alpha} v$,

$\Sigma \pi \varepsilon \rho \chi \varepsilon 10 \hat{~} \tau \varepsilon \pi \alpha \rho$ ' ö $\chi \theta \alpha \varsigma^{1}$.

On en déduira que les Maliades se définissent comme des nymphes et que, d'autre part, leur nom pourrait être un dérivé en - $\alpha \delta$ - du nom divin $M \alpha \lambda i i_{\text {; }}$; cette dernière est à considérer comme une nymphe, une déesse des sources et de l'eau, comme il ressort d'ailleurs d'un passage de Théocrite ${ }^{2}$. Or, j'avais récemment évoqué le lien possible unissant Ma $\lambda$ ís et la divinité anatolienne Maliya plusieurs fois citée dans les textes hittites; Maliya y apparaît nettement comme une déesse liée à l'eau, protectrice des jardins et des vignobles ${ }^{3}$.

1 SOPHOCLE, Philoctète, 723-725:

*... (qui le) conduit vers la demeure paternelle,

(séjour) des nymphes Maliades,

et le long des rives du Sperchéios».

Pour les divinités Maliades, cf. Roscher, Lexikon, p. 2304.

2 THÉOCRITE, Idylles, XIII,

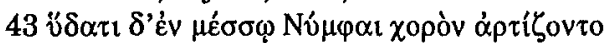

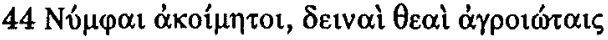

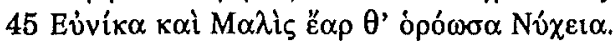

43 «Au milieu de l'eau des Nymphes formaient un chœur,

44 des Nymphes ignorant le sommeil, divinités terribles pour les campagnards,

45 Eunica ainsi que Malis et Nycheia contemplant le printemps.»

Pour les dérivés en - $\alpha \delta$ - du type $M \alpha \lambda$ tó $\delta \varepsilon \zeta$, cf. P. Chantraine, La formation des noms en grec ancien, Paris, 1979 (nouveau tirage), p. 354; un excellent parallele nous est offert avec $\Delta \rho \nu \alpha \dot{\delta} \delta \varepsilon \varsigma(v o ̛ ́ \mu \varphi \mathrm{l})$ ), dérivé de $\delta \rho \hat{\jmath} \zeta$; le mot dérivé vauit comme adjectif et substantif et met en évidence le lien, le rapport ou la ressemblance existant entre le dérivé et le nom à l'origine de celui-ci.

3 Cf. R. LEBRUN, Maliya, une divinité anatolienne mal connue, in Studia Paulo Naster oblata II, Louvain, 1982, p. 124 et 126-128. Pour Malis, cf. Roscher, Lexikon, p. 2305 et O. MASSON, Les fragments du poete Hipponax, Paris, 1962, p. 128 sq. Malis était notamment vénérée en Isaurie et en Lydie où elle était d'ailleurs la grande déesse de Dalisandos. 
La divinité ${ }^{\mathrm{d}}$ Maliya/d ${ }^{\mathrm{d}}$ Maliya ${ }^{4}$ est elle-même à l'origine du dérivé pluriel dMaliyanni désignant manifestement les «nymphes» suivantes de la déesse Maliya.

Dès lors, un parallélisme significatif s'établit entre l'anatolien Maliya-

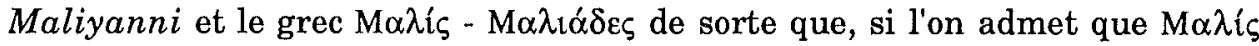
continue la divinité hittite Maliya, les $M \alpha \lambda \downarrow \alpha ́ \alpha \varepsilon \varsigma \zeta$ apparaissent comme la continuité, dans le cadre grec, des Maliyanni. Il n'est pas inutile de repréciser ici que Maliya/Malis était encore vénérée dans plusieurs cités de Lycie aux Ve et IVe s. av. J.-C. en tant que divinité poliade, ce qui explique son assimilation à Athéna (cf. les inscriptions en langue lycienne) ${ }^{5}$.

A l'heure où l'on réexamine le problème des contacts entre le monde anatolien et les gens de l'Ahhiyawa (Achéens) durant la seconde moitié du second millénaire avant J.-C. 6 , et quand on revoit l'introduction d'entités divines issues du Proche-Orient dans la famille religieuse grecque (par ex.

$4 \quad$ id Maliya $=$ la rivière Maliya; ${ }^{\mathrm{d}}$ Maliya $=$ la divinité Maliya.

5 Pour les mentions de Maliya dans les textes lyciens, cf. R. LEBRUN, art. cit., p. 124, 129,130 .

6 L'archéologie atteste clairement la présence mycénienne sur les côtes de l'Asie Mineure égéenne ainsi que sur celles de l'Anatolie méridionale; Milet en est un bon exemple. Sans entrer ici dans l'historique détaillé des discussions, disons simplement que ces relations entre les Hittites et les Mycéniens se clarifient par les textes hittites si on admet l'équation hitt. Ahhiyawa $=$ Grecs Achéens. Déjà en 1924, E. FORRER avait avancé une telle hypothèse dans $M D O G, 63$ (1924), p. 1-22 et dans $O L Z, 27$ (1924), p. 113-118; il s'était vu néanmoins contré plus tard par F. SOMMER dans sa magistrale étude qui réunissait la documentation concernant l'Ahhiyawa, à savoir Die Ahhijava-Urkunden, Munich, 1932. Depuis quelques années le débat a été relancé avec force et l'identification des gens de l'Ahhiyawa avec les Achéens - à laquelle je me rallie - gagne du terrain. Bien qu'une localisation précise de l'Ahhiyawa demeure difficile, il semble que le terme désigne une zone de l'Anatolie occidentale ayant accès à la mer (et pouvant exclure la Troade) et que, par extension, il désigna progressivement pour les Hittites l'ensemble des territoires occupés par des Achéens, ce qui explique que le roi de l'Ahhiyawa était considéré comme l'égal du grand roi hittite. A titre indicatif, voici quelques études importantes et récentes liées au problème de l'Ahhiyawa: G. STELNER, Die Ahhijawa-Frage heute, in Saeculum, 15 (1964), p. 365-392 (pour lui, l'Ahhiyawa est une puissance anatolienne); H.G. GÜterbock, The Hittites and the Aegean world: 1. The Ahhiyawa Problem Reconsidered, in AJA, 87 (1983), p. 133 sq.; ID., Hittites and Akhaeans : a new look, in PAPhS, 128, 2 (1984), p. 114-122; ID., Troy in Hittite Texts? Wilusa, Ahhiyawa and Hittite History, in Symposium on the Trojan War held at Bryn Mawr College, oct. 1984, 1986, p. 33-44; I. SINGER, Western Anatolia in the 13th Century B.C. according to the Hittite Sources, in AS, XXXIII (1983), p. 205-217. Précisons encore qu'il ne s'agit ici que d'un choix de quelques études solides susceptibles d'orienter correctement l'historien dans la problématique. 
Aphrodite, Artémis) ${ }^{7}$, le parallélisme qui vient d'être évoqué constitue un nouvel élément révélateur des conséquences religieuses et cultuelles de ces contacts noués à l'époque mycénienne entre l'Anatolie occidentale et le monde achéen, à moins qu'il ne faille, dans le cas qui nous occupe, rabaisser la date à l'établissement des colonies grecques d'Asie Mineure et de la progressive hellénisation de celle-ci.

\section{Zeus Osogoa ${ }^{8}$}

Voici près d'un demi-siècle, A. Namitok publiait dans la Revue de l'Histoire des Religions un remarquable article consacré à Zeus Osogoa, un dieu de l'orage dont l'épiclèse s'avère clairement anatolienne ${ }^{9}$.

Les données essentielles étaient les suivantes. Zeus Osogoa, divinité particulière de la tribu des Otorcondes, était le grand dieu de Mylasa, ville de Carie où il possédait son sanctuaire ${ }^{10}$. Il fut assimilé à Jupiter Dolichenus et, comme souvent en Asie Mineure, l'épiclèse suffit à elle seule pour désigner le dieu tant elle en est caractéristique ${ }^{11}$. Les inscriptions attestent la venue dans le sanctuaire carien de pèlerins depuis les zones contiguës de Lycie et de Pamphylie ${ }^{12}$.

Le caractère anatolien de Zeus Osogoa s'impose d'autant plus qu'il est vénéré par les Cariens méridionaux, les Lyciens et les Pamphyliens, c'est-àdire par des populations issues de régions où la tradition religieuse louvite est demeurée particulièrement vivace. Dans le cadre du syncrétisme grécoanatolien, le Zeus qui nous occupe, se réfêre, sous habillage grec, au dieu de l'orage louvite Tarchunt, "le Victorieux", retrouvé maintes fois dans les inscriptions lyciennes des Ve et IVe s. av. J.-C. (lyc. Trqqñt dans l'anthroponymie gréco-asianique de Lycie, Pamphylie, Cilicie et Carie ${ }^{13}$.

7 Artémis me semble être un des noms de ces importantes divinités désignées usuellement dans les textes hittites par le sumérogramme dKAL, à savoir les divinités protectrices de la nature sauvage. Le lycien Ertemi- continue bien le nom anatolien et ne me semble pas être un emprunt au grec. Cf. R. LEBRUN, Problemes de religion anatolienne in Hethitica ,VIII (1987), p. 251, 252, 260.

8 Pour Zeus Osogoa, cf. Real-Encyclopedie, p. 1585-1587.

9 Cf. A. NAMITOK, in RHR, 124 (1941), p. 97-109.

10 La ville de Mylasa est située à l'intérieur des terres, sur la hauteur. Cf. L. ZGUSTA, Kleinasiatische Ortsnamen, Heidelberg, 1984, p. 406, § 861, 1.

11 Ainsi, sur les plus anciennes monnaies de la cité.

12 Cf. Le BAS-WADDINGTON, $n^{\circ} 349$ à 358.

13 Cf. Ph.H.J. Houwink ten CATE, The Luwian Population Groups of Lycia and Cilicia Aspera during the Hellenistic Period, Leyde, 1961, p. 125-128. 
A. Namitok, avec les possibilités de l'époque, s'était risqué à une explication de l'épiclèse Osogoa par le hatti, la langue des Pré-hittites, en reconnaissant dans Osogoa la grécisation du hatti wašḩa $(w)$, «dieu». L'auteur avait raison de chercher une interprétation de l'épithète divine dans le monde anatolien, mais, de façon fort compréhensible, il se trompait de piste. Il apparaît aujourd'hui que, pour la majorité des épiclèses indigènes des régions citées dans cette contribution, la solution doit être recherchée dans la langue louvite (désormais assez bien connue) et ses prolongements au sein du premier millénaire avant notre ère.

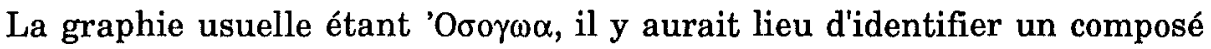
du louvite wasu- «bon» + le thème louvite kuwa-. En effet, dans le seul cadre du louvite, il est possible d'envisager une réduction de wasu- en *usu- si l'on se réfère aux alternances à l'initiale de type identique offertes par wastul/ustul, «faute» ou encore wanati-/unati- «femme», Le passage du louvite $u$ à la notation grecque $o$ est régulier. Quant à l'élément $-\gamma \omega \alpha$, il pourrait être rattaché au thème kuwa- bien représenté dans l'onomastique hittito-louvite. Il apparaît en seconde position dans plusieurs noms propres $\mathrm{d}^{\text {'Anatolie méridionale }}{ }^{14}$. Le sens et la valeur du radical $k u w a$-demeurent obscurs. Toutefois, abstraction faite de l'onomastique, un radical louvite $k u w a-$ se retrouve de façon significative notamment dans kuwaya-, "craindre» et ses dérivés, dans les substantifs kuwala-, «armée» et kuwatna"armée, camp», dans l'adjectif kuwanzu(i)- qui détermine le terme "dieux» (cf. E. LAROCHE, Dictionnaire de la langue louvite, Paris, 1959, p. 58-60 et M. POETTO, Kadmos, 21, 2 [1982], p. 101-103). De plus, si, suivant une note récente de F. Starke $(K Z, 100,2$. Heft [1987], p. 256, n. 50a), on admet que ${ }^{\mathrm{f}} M a n a-k u w a n z u i s$ est la lecture du nom propre féminin noté ${ }^{\mathrm{f}} M a-n a-$ DUGUD-iš en KUB XL 83 Ro 3', 21', il y aurait confirmation d'un lien unissant le thème kuwa-> gr. $\gamma \omega \alpha_{-}, \kappa \omega \alpha_{-}^{-}, \gamma \circ \alpha_{-}, \kappa \circ \alpha_{-}$, et le sumérien DUGUD signifiant usuellement «important, puissant». Il résulte de ces considérations que le radical kuwa-contiendrait l'idée de puissance, de force (parfois terrifiante).

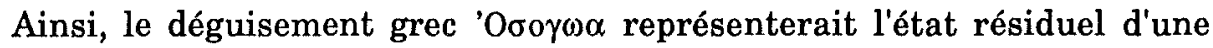
épiclèse louvite *wasu-kuwa- soulignant l'aspect bienfaisant et puissant du grand dieu de l'orage, ce que mettaient parfois en évidence d'autres épithètes anatoliennes mieux connues.

14 Cf. Ph.H.J. HouwINK ten CATE, op. cit., p. 152-153; E. LAROCHE, Les noms des Hittites, Paris, 1966, p. 101-103. 


\section{Artémis Pérasia}

Strabon désigne l'Artémis Pérasia comme la grande déesse d'Hiérapolis Castabala en Cilicie, une divinité dont le culte était assuré par des prêtresses marchant pieds nus sur des charbons sans éprouver la moindre douleur ${ }^{15}$. Il s'agit, en fait, d'un culte indigène comme le révèle l'épiclèse de la déesse.

En effet, le terme Pérasia n'a reçu à ce jour aucune explication convaincante. Or, il me semble que l'Artémis Pérasia de la période gréco-asianique constitue la continuité de l'Ishtar parassi hittite/louvite.

a) Le texte de l'Apologie du roi hittite Hattusili III (1285-1250 av. J.-C.) fait référence à une Ishtar parassi- "Ištar/Š́auška de la promesse»16. Cette épithète attribuée à l'Ishtar hourrite constitue le dérivé adjectival régulier louvite en -assi- du thème nominal para-, pra- répondant vraisemblablement au sens de "promesse». Les règles phonétiques d'évolution du louvite vers la période gréco-asianique permettent d'affirmer que la forme parassi aboutit logiquement à peras(s)i-/paras(s)i- assorti, sous déguisement grec, d'une voyelle thématique $-a$-pour caractériser le féminin de l'épiclèse ${ }^{17}$.

b) La déesse Shaushka, forme hourrite de l'Ishtar babylonienne, a connu, surtout à partir du XIIIe s. av. J.-C., un franc succès en Anatolie méridionale ${ }^{18}$. Elle va toutefois se trouver progressivement supplantée par Kubaba (Cybèle), la grande déesse de Kargémish, comme l'attestent d'ailleurs les inscriptions louvites hiéroglyphiques de Cilicie. De plus, en certains lieux, se réalisera l'assimilation de Kubaba à Artémis comme le signale Hésychius et le souligne L. Robert ${ }^{19}$. Il existe ainsi une continuité entre Ishtar/Shaushka, Kubaba et Artémis pour laquelle d'ailleurs une origine anatolienne n'est pas à exclure ${ }^{20}$.

15 Strabon, Géogr., XII, 537.

16 Apologie de Hattusili III, col. IV, 15 ...zi-iq-qa-wa-mu-za 16 d ${ }^{2}$ ŠTAR pa-ra-aš-ši-in i-ya "quant à toi fête-moi comme Ishtar de la promesse".

17 Parassi-paraît devoir être analysé comme un adjectif de relation en -(a)ssi- de paraI pra-, adjectif qui, en louvite, tient lieu de génitif. Pour le sens du thème nominal para/pra-, cf. F. STARKE, in KZ, 100 (1987), p. 253, n. 38.

18 Cf. R. LEBRUN, Samuha foyer religieux de l'Empire hittite, Louvain-la-Neuve, 1976, passim.

19 Cf. L. Robert in A. Dupont-Sommer et L. Robert, La déesse de HiérapolisCastabala, Paris, 1964, p. 14.

20 Cf. note 6. Artémis est chez elle en Asie Mineure et il est d'autant plus significatif de la voir protéger les Troyens (probablement une population à majorité hittito-louvite), en compagnie de son frère Apollon (d'origine proche-orientale ?) et d'Aphrodite, dans le conflit opposant ces derniers aux Achéens. 
c) Il se pourrait, dès lors, qu'au second millénaire Ishtar parassi ait été une divinité importante adorée dans la région de Castabala. A travers quelques avatars, son culte s'est maintenu en ce lieu et c'est cette même déesse que nous retrouvons, sous déguisement grécisé, dans l'Artémis Pérasia. Il s'agit ici d'un nouvel exemple de continuité religieuse.

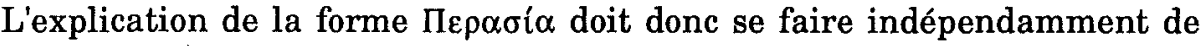
l'araméen $P w s ̌ r$ pour lequel les explications n'étaient guère convaincantes. La fausse étymologie de Strabon ${ }^{21}$ est aussi à rejeter au même titre que celle de certains modernes voyant dans Artémis Pérasia une forme aberrante de

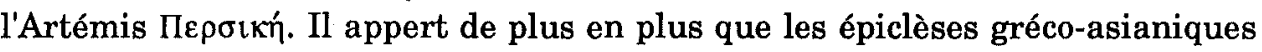
doivent être étudiées méthodiquement, en elles-mêmes, et dans une perspective de continuité linguistico-religieuse louvite.

Institut Catholique de Paris

René LEBRUN

Rue d'Assas, 21

F - 75006 PARIS

et

Katholieke Universiteit Leuven

Blijde Inkomststraat, 21

B - 3000 LEUVEN

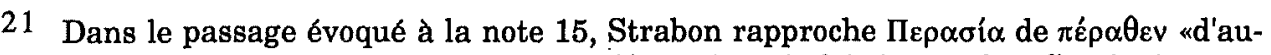
delà»; ainsi, le sens précis de l'épithète d'Artémis était déjà ignoré dans l'antiquité. 